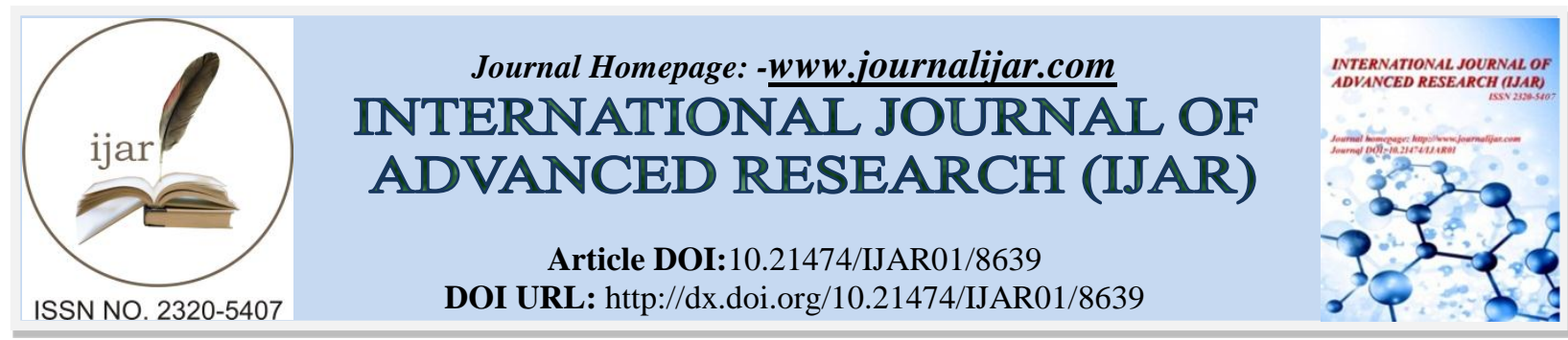

RESEARCH ARTICLE

\title{
ETUDE PHYTOCHIMIQUE ET ACTIVITES BIOLOGIQUES DE DEUX PLANTES : ALCHORNEA FLORIBUNDA (EUPHORBIACEAE) ET BRIDELIA FERRUGINEA (PHYLLANTHACEAE).
}

\section{Boris Achille Eyi Mintsa ${ }^{1}$, Ibrahim ${ }^{1}$, Rolande Mabika Mabika ${ }^{2}$, Wenceslace Landry Mve Mendame ${ }^{1}$, Alexis Nicaise Lepengue $^{2,3}$, Alain Souza1 ${ }^{1,2}$ and Jean Fabrice Yala ${ }^{2}$.}

1. Laboratoire de Physiologie animale et Pharmacologie, Unité de recherche Agrobiologie, Université des Sciences et Techniques de Masuku (USTM), BP 067 Franceville, Gabon.

2. Laboratoire de Biologie Moléculaire et Cellulaire, équipe de Bactériologie-Immunologie, Unité de recherche Agrobiologie, Université des Sciences et Techniques de Masuku (USTM), BP 067 Franceville, Gabon.

3. Laboratoire de Physiologie végétale, Phytopathologie et Amélioration des plantes, Unité de recherche Agrobiologie, Université des Sciences et Techniques de Masuku (USTM), BP 067 Franceville, Gabon.

\section{Manuscript Info}

\section{Manuscript History}

Received: 08 January 2019

Final Accepted: 10 February 2019

Published: March 2019

Key words:-

Diarrhea; Alchornea floribunda; Bridelia ferruginea; DPPH; Medicinal plants; Phytochemical screening.

\section{Abstract}

In addition to metabolic diseases, microbial pathologies often characterized by resistance and multiresistance phenomena of certain strains with known reference molecules, constitute a real public health problem in the world as a result of the number of deaths caused each year by these pathologies.

The purpose of this work is to evaluate the antioxidant and antimicrobial potential of aqueous extracts of Alchornea floribunda and Bridelia ferruginea.

The phytochemical composition of the extracts was determined by the calorimetric and differential method. The antioxidant activity was evaluated by the antiradical activity of the radical DPPH. Also, antimicrobial activity was determined by the well method in agar and determination of minimum inhibitory (MIC) and bactericidal (MBC) concentrations.

The results of the phytochemical screening reveal a wealth of phytomolecules with antimicrobial properties such as polyphenols, terpenes, flavonoids, tannins and alkaloids. Also, they emphasize that both extracts have antioxidant activities. However, the average antioxidant activity of the extract of Alchornea floribunda is greater than that of Bridelia ferruginea. This activity is comparable to that of gallic acid and ascorbic acid in the DPPH free radical scavenging assay.

The study of the antimicrobial activity of these two plants revealed a significant antibacterial activity with respect to several bacterial strains responsible for infantile diarrhea, namely: Shigella sonnei, Shigella spp., Yersinia pestis, Raoultella ornithinilytica, Escherichia vulneris, Salmonella choleraesuis, Escherichia coli, as well as a remarkable yeast antifungal activity (Candida spp PV, Candida albicans bucal, Candida albicans $\mathrm{PV})$. 


\section{Introduction:-}

Les maladies métaboliques (cancers, hypertension, diabète, insuffisance cardiaque, asthme, Alzheimer) constituent un véritable problème de santé mondiale suite au nombre de décès causés chaque année (OMS, 2013). Elles ont pour cause commune un certain nombre de facteurs tels que : le vieillissement, la sédentarité, le tabagisme, l'usage nocif de l'alcool et les carences en vitamines dues à une mauvaise alimentation. Près de $80 \%$ des décès sont dus aux maladies non transmissibles, soient 29 millions d'individus dans les pays à faible revenu et plus de 9 millions de ces décès surviennent avant l'âge de 60 ans (OMS, 2013).

L'oxygène est essentiel pour tous les organismes eucaryotes. Cependant, même dans les réactions métaboliques fondamentales se produisant dans les cellules, les formes réactives de l'oxygène sont capables d'endommager diverses biomolécules telles que les protéines, l'acide désoxyribonucléiques (ADN) ou des acides gras polyinsaturés (Gruber, Schaffer, and Barry, 2008). En plus de la production normale des radicaux libres dans les cellules durant la respiration et le métabolisme, les quantités excessives des espèces réactives oxygènes (ERO) peuvent se former et leurs taux sont élevés durant le stress oxydatif en raison de l'inflammation, l'activité physique intense, l'ischémie ou un traumatisme (Cardona et al., 2013; Lahouel et al., 2004; Pincemail et al., 2007; Shoji et al., 2004). La carence en substances nutritives protectrices nécessaires ou d'autres substances antioxydantes dans le régime alimentaire ou dans certaines conditions héréditaires pathologiques peuvent aussi affecter négativement l'équilibre entre les prooxydants et les antioxydants dans l'organisme (Cardona et al., 2013; Lahouel et al., 2004; Pincemail et al., 2007; Shoji et al., 2004).

A côté des maladies métaboliques, les maladies infectieuses représentent également un problème de santé publique majeur à cause de leur ampleur (OMS, 2013). En effet, le phénomène de résistance et de multirésistances de certaines souches microbiennes aux antibiotiques de références et communément utilisés augmente le taux de mortalité engendré par des pathologies telles que : le paludisme, le VIH-Sida, la tuberculose, l'Ebola, les diarrhées infectieuses (OMS, 2013). Les maladies diarrhéiques sont et restent une source de mortalité et de morbidité en santé publique dans le monde avec plus de 2 millions de décès par année (Bonkoungou et al., 2013; Clarke, 2001), et touchent particulièrement les enfants de moins de 5 ans (Kosek, 2003). Il a été récemment estimé que les diarrhées comptent pour 9,9\% des 6,9 millions des décès des enfants de moins de 5 ans en 2011 (Liu et al., 2012; Walker, 2012). Cependant, dans les pays pauvres (PP) et les pays en voie de Développement (PED) les taux de maladies diarrhéiques sont très élevés. Effectivement, elles sont responsables de 16 à $35 \%$ des décès des enfants de moins de 5 ans (Kosek, 2003). Cette pathologie négligée représente la troisième cause de décès des maladies infectieuses mondiale (OMS, 2011). En Afrique, particulièrement au Gabon, les premières prises en charge dans les zones rurales se font par l'administration des décoctions des plantes.

Aussi, l'Organisation Mondiale de la Santé (OMS) estime qu'en Afrique, plus de $80 \%$ de la population utilisent encore les plantes médicinales pour répondre à leurs besoins de santé primaire (OMS, 2002). Cette utilisation des plantes remonte à la préhistoire par l'homme pour satisfaire ses besoins nutritionnels et thérapeutiques à cause de leur richesse en métabolites secondaires (Konaté et al., 2011; Nsi AKoué et al., 2013). Le Gabon ne fait pas exception, car l'utilisation des plantes médicinales pour le traitement de différentes affections se fait depuis des générations (Mengome, 2013).

Le Gabon, situé en Afrique Centrale, est couvert à $85 \%$ d'une forêt équatoriale humide, soit $12 \%$ des forêts du bassin du Congo (Devers et al., 2006). Ces forêts gabonaises renferment une importante réserve d'espèces animales et végétales, dont une large proportion reste endémique. Son couvert végétal compte environ 10000 espèces inventoriées, constituant ainsi un important réservoir de molécules naturelles (Devers et al., 2006). Plusieurs travaux ont été mené dans le souci de valoriser le potentiel nutritionnel et thérapeutique des espèces végétales du Gabon.

A cet effet, les travaux de Ondo et al. (Ondo et al., 2013) ont montré que Asplenium africanum (Aspleniaceae) et les fruits de Megaphrinium macrostachyum (Marantaceae) contenant les phénols avaient une activité antioxydante. Nsi et al. (Nsi Akoué et al., 2013) ont révélé que Sakersia africana, plante utilisée dans le régime alimentaire par les mandrills du parc de la Lékédi à Bakoumba (Haut-Ogooué) avait une activité antiradicalaire et antioxydante. Par ailleurs, Eyi Mintsa et al. (Eyi Mintsa, 2017) ont montré que les feuilles de Helichrysium mechowianum utilisées par les pygmées du Gabon avaient des propriétés oestrogéniques pour le raffermissement de l'appareil génital (utérus) féminin après accouchement. De plus, Yala et al. (Yala et., 2016) ont montré que l'extrait aqueux de Eryngium foetidium récolté à Franceville présentait une activité antibactérienne. Aussi, Nsi Akoué (Nsi Akoué, 2017) a révélé 
le pouvoir antimicrobien des plantes consommées par les mandrills, à l'exemple de Medinilla mirabilis, Pentaclethra eetveldeana, Berlinia bracteosa et Tristema mauritianum, qui inhibent la croissance des souches bactériennes telles que : Eschérichia coli, Enterobacter cloacae.

Ce travail fait suite à ceux de Nsi Akoué en 2017 qui ont identifié les plantes consommées par les mandrills du parc de la Lékédi Bakoumba et le choix des plantes médicinales s'est fait par l'approche Zoopharmacognosie. Cette approche porte sur les comportements alimentaires des mammifères notamment les grands singes qui font usage de certaines espèces végétales, pour améliorer leur état de santé (Huffman, 2003; Nsi Akoué, 2017).

Ainsi, les deux plantes utilisées dans cette étude font partie du régime alimentaire des mandrills (Mandrillus sphinx) du parc de la Lékédi à Bakoumba. C'est dans ce contexte que l'objectif général de cette étude s'est focalisée d'évaluer les activités biologiques de deux plantes à savoir : Alchornea floribunda (Euphorbiaceae) et Bridelia ferruginea (Phyllanthaceae).

Les objectifs spécifiques visent à : mettre en évidence par un Screening phytochimique les molécules chimiques ayant des propriétés pharmacologiques, évaluer l'activité antioxydante et antimicrobienne de ces extraits de plantes sur les bactéries isolées des diarrhées infantiles et sur les souches de Candida.

\section{Matériel Et Méthodes:- \\ Matériel végétal}

Le matériel végétal utilisé était constitué des graines de Alchornea floribunda et des écorces de Bridelia ferruginea. Ces plantes sont issues d'une campagne d'échantillonnage au parc de la Lékédi à Bakoumba dans le Département de la Lékoko, dans la province du Haut-Ogooué (Nsi Akoué, 2017).
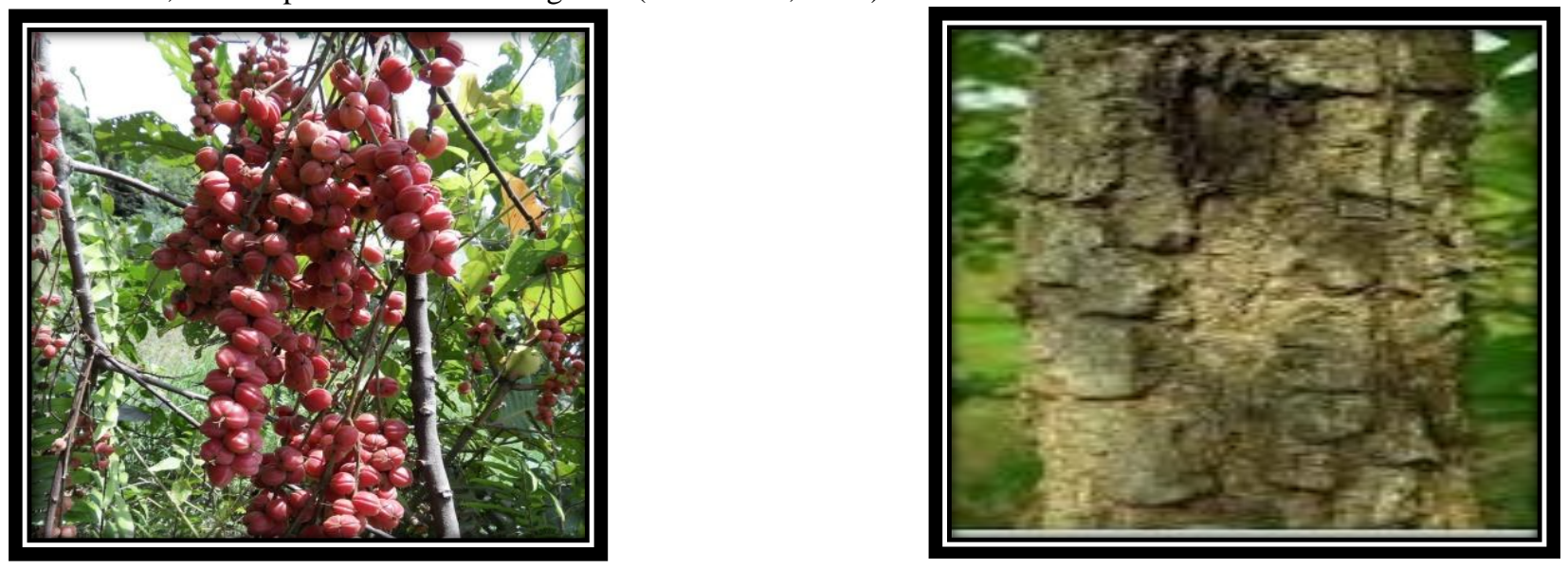

Figure1. Matériel végétal utilisé. A : les graines de Alchornea floribunda Müll. Arg ; B : les écorces de Bridelia ferruginea Benth

\section{Préparation des extraits végétaux}

Les parties des plantes récoltées et identifiées ont été séchées au laboratoire, à la température ambiante et à l'abri de la lumière. Une fois séchées, les graines et les écorces de ces plantes ont été broyées à l'aide d'un broyeur de type Waring-R commercial ARMOR jusqu'à l'obtention des poudres. Les poudres ont été macérées sous agitateur magnétique pendant 24 heures. Le macérat obtenu a été ensuite filtré à l'aide du papier filtre, puis lyophilisée, et lyophilisat obtenu a été conservé dans des bocaux à $4{ }^{\circ} \mathrm{C}$ jusqu'à la réalisation des tests.

\section{Screening phytochimique}

Le criblage phytochimique a permis de mettre en évidence la composition des métabolites secondaires présents dans les plantes. Ainsi, $5 \mathrm{~g}$ de poudre de matière végétale ont été mélangées à $250 \mathrm{~mL}$ d'eau distillée, puis le mélange a été porté à ébullition pendant 15 minutes. Le décocté obtenu a été filtré à l'aide d'un coton. Pour chaque test, $2 \mathrm{~mL}$ du filtrat ont été utilisés. 
Les essais phytochimiques des extraits aqueux ont été réalisés selon les techniques classiques décrites par Paris (Paris and Moyse, 1969), Bouquet (Bouquet and Fouret, 1975; Bouquet, 1971). Ces essais ont pour objectifs de mettre en évidence les groupes chimiques ayant des propriétés pharmacologiques.

\section{Test des saponosides}

Deux millilitres $(2 \mathrm{~mL})$ de l'extrait de plante ont été versés dans un tube à essai, puis le tube a été agité vigoureusement. 5 minutes après, la présence d'une mousse persistante met en évidence la présence des saponosides.

\section{Test des tannins}

Deux millilitres $(2 \mathrm{~mL})$ de l'extrait de plante ont été versés dans un tube, puis $2 \mathrm{~mL}$ du trichlorure de fer $(\mathrm{FeCl} 3)$ à 1 $\%$ ont été ajoutés. Lorsque le mélange donne une coloration verdâtre ou bleu noirâtre, nous avons la présence des tannins.

\section{Test des polyphénols}

Deux millilitres $(2 \mathrm{~mL})$ de l'extrait de plante ont été transférés dans un tube, ensuite $1 \mathrm{~mL}$ du réactif de Folin Ciocalteu (RFC) et $1 \mathrm{~mL}$ de bicarbonate de sodium $\left(\mathrm{Na}_{2} \mathrm{CO}_{3}\right)$ ont été ajoutés. Lorsque le mélange donne une coloration vert foncé, cela indique la présence des polyphénols.

\section{Test des flavonoïdes}

Deux millilitres $(2 \mathrm{~mL})$ de l'extrait de plante ont été mis dans un tube, puis $1 \mathrm{~mL}$ d'acide chlorhydrique et $1 \mathrm{~mL}$ de bicarbonate de sodium $\left(\mathrm{Na}_{2} \mathrm{CO}_{3}\right)$ ont été ajoutés. L'ajout de l'acide entraine l'apparition d'une coloration foncée et l'ajout de la base accentue la coloration.

\section{Test des alcaloïdes}

Les alcaloïdes ont été mis en évidence par le réactif de Mayer. L'ajout de quelques gouttes de ce réactif à $2 \mathrm{~mL}$ de la solution d'extrait de plante entraîne la formation d'un précipité rouge orangé en présence des alcaloïdes.

\section{Test des composés Anthracéniques}

Deux millilitres $(2 \mathrm{~mL})$ de l'extrait de plante ont été transférés dans un tube et $2 \mathrm{~mL}$ d'hydroxyle d'ammoniaque $\left(\mathrm{NH}_{4} \mathrm{OH}\right)$ ont été ajoutés et le tube a été agité. Lorsque le mélange donne une coloration rouge, nous avons la présence des composés anthracéniques.

\section{Test des stérols/ terpènoïdes}

Deux millilitres $(2 \mathrm{~mL})$ de l'extrait de plante ont été mis dans un tube et $2 \mathrm{~mL}$ d'acide sulfurique $\left(\mathrm{H}_{2} \mathrm{SO}_{4}\right)$ ont été ajoutés. L'apparition d'un anneau rouge brun ou violet, indique la présence des stérols ou des terpènoïdes.

\section{Test des hétérosides cardiotoniques}

Deux millilitres $(2 \mathrm{~mL})$ de l'extrait de plante ont été mis dans un tube et $1 \mathrm{~mL}$ d'acide sulfurique et $1 \mathrm{~mL}$ de sulfate ferrique ont été ajoutés. Lorsque le mélange donne une coloration brune sale, l'extrait contient la digitoxine. Si la coloration est rouge fluorescent, l'extrait contient la digitoxigenine. Lorsque la coloration est jaune puis vire au rouge bleu, l'extrait contient la gitoxine et si la coloration est jaune puis vire au rouge violet l'extrait contient la gitoxigenine.

\section{Test des sucres réducteurs}

Deux millilitres $(2 \mathrm{~mL})$ de l'extrait de plante ont été transférés dans un tube, puis $2 \mathrm{~mL}$ de liqueur de Fehling ont été ajoutés. Le tube a été chauffé jusqu'à ébullition, puis refroidit. L'apparition d'un précipité rouge brique, révèle la présence des sucres réducteurs.

\section{Evaluation de l'activité antioxydante par la méthode de l'index}

L'index d'activité antioxydant (IAA) a été déterminé par la méthode décrite par Scherer et Godoy (Scherer and Godoy, 2009). Cette méthode est basée sur le test du 2,2-diphényl-1-picrylhydrazyle (DPPH) c'est-à-dire la capacité à réduire le radical libre DPPH.

Pour cette étude, l'évaluation a été réalisée pour une gamme de concentration de l'extrait aqueux allant de 5 à 50 $\mu \mathrm{g} \cdot \mathrm{mL}^{-1}$ obtenue par dilution progressive à partir d'une solution mère de $100 \mu \mathrm{g} . \mathrm{L}^{-1}$. Ainsi, $100 \mu \mathrm{L}$ de chaque 
dilution ont été mélangés avec $100 \mu \mathrm{L}$ de DPPH. La solution de DPPH a été préparé à une concentration de 100 $\mu \mathrm{g} . \mathrm{L}^{-1}$ dans l'éthanol. Le mélange a été agité vigoureusement et laissé au repos à la température du laboratoire pendant 15 minutes. Les absorbances ont été mesurées à une longueur d'onde de 517 nm à l'aide d'un spectrophotomètre. L'acide ascorbique et l'acide gallique ont été utilisés comme références.

$$
A A(\%)=\left(\frac{\text { Abs }(\text { control })-\text { Abs }(\text { Echantillon })}{A b s(\text { control })}\right) \times 100
$$

AA : activité antioxydante ; Abs control : absorbance du control ; Abs extrait : absorbance de l'extrait Les concentrations inhibant 50\% de DPPH (IC50) de l'extrait et des standards ont été déterminées en utilisant la droite de régression linéaire. L'index d'activité antioxydant a été calculé par la formule suivante :

$$
\boldsymbol{I A A}(\%)=\frac{[\boldsymbol{D P P H}]}{\boldsymbol{I C} 50} \text { Finale }
$$

Selon l'échelle d'évaluation de Scherer et Godoy (2009), l'activité antioxydante d'un extrait de plante peut être caractérisé par rapport à l'indice d'activité antioxydante (IAA). Elle est dite faible pour IAA $<0,5$, modérée pour $0,5<\mathrm{IAA}<1$, forte pour $1<\mathrm{IAA}<2$ et très forte IAA $>2$.

\section{Evaluation de l'activité antimicrobienne Matériel microbien}

Le matériel microbiologique utilisé a été fourni par le Laboratoire de Biologie Moléculaire et Cellulaire (LABMC). Ce matériel était constitué de dix souches bactériennes isolées des fèces diarrhéiques des enfants de 0-5 ans et de quatre souches fongiques isolées des patients à l'Hôpital Militaire Omar Bongo Ondimba de Libreville, tableau 1.

Tableau 1:-Les souches microbiennes

\begin{tabular}{|l|c|l|}
\hline Familles & Genres & Espèces \\
\hline Enterobacteriaceae & Salmonella & choleraesuis \\
\cline { 2 - 3 } & Salmonella & parathyphie A \\
\cline { 2 - 3 } & Serratia & ordorifera \\
\cline { 2 - 3 } & Klebsiella & oxytoca \\
\cline { 2 - 3 } & Shigella & sonnei \\
\cline { 2 - 3 } & Raoultella & ornithinolytica \\
\cline { 2 - 3 } & Shigella & pestis \\
\cline { 2 - 3 } & Yersinia & vulneris \\
\cline { 2 - 3 } & Escherichia & soli \\
\hline Saccharomycetaceae & Escherichia & albicans PV \\
\cline { 2 - 3 } & Candida & albicans \\
\cline { 2 - 3 } & Candida & spp PV \\
\cline { 2 - 3 } & Candida & \\
\cline { 2 - 3 } & Candida & \\
\hline
\end{tabular}

PV : prélèvement vaginal

\section{Préparation des milieux de cultures}

Trois types de milieux de culture ont été utilisés respectivement pour la culture microbienne et les tests antimicrobiens : le milieu cœur-cervelle (BHI-D), la gélose Sabouraud complémentée avec du Chloramphénicol 2 (SAB CHL-2) et la gélose Mueller Hinton (MH). Ces milieux de cultures ont été autoclavés à $121^{\circ} \mathrm{C}$ pendant 15 minutes.

\section{Préparation des extraits}

Cent milligrammes (100 $\mathrm{mg}$ ) d'extraits aqueux de A. floribunda et de B. ferruginea ont été pesés et introduit dans les tubes Eppendorf. Ces extraits ont été solubilisés avec $1 \mathrm{~mL}$ d'un mélange hydro-éthanolique contenant $750 \mu \mathrm{L}$ d'eau distillée stérile et $250 \mu \mathrm{L}$ d'éthanol à $70 \%$, puis vortexé avant d'être utilisé pour les différents tests. 


\section{Préparation des cultures microbiennes}

Une à deux colonies bactériennes ont été délayées dans $8 \mathrm{~mL}$ de milieu $\mathrm{BHI}$ liquide, puis le tout a été incubé à $37^{\circ} \mathrm{C}$ pendant $18 \mathrm{~h}$ à $24 \mathrm{~h}$. Ensuite, $10 \mu \mathrm{L}$ de cette pré-culture ont été ensemencées par la méthode de stries sur la gélose $\mathrm{BHI}$, et les boîtes ont été incubées à $37^{\circ} \mathrm{C}$ pendant $18 \mathrm{~h}$ à $24 \mathrm{~h}$. Les colonies jeunes de $18 \mathrm{~h}$ à $24 \mathrm{~h}$ obtenues serviront à la préparation des inocula.

Les cultures fongiques ont été effectuées par ensemencement par la méthode de stries sur gélose Sabouraud inclinée supplémenté au Chloramphénicol 2 (SAB CHL 2-D) à partir des stocks cryoconservés, puis incubées à $37^{\circ} \mathrm{C}$ pendant $18 \mathrm{~h}$ à $24 \mathrm{~h}$.

\section{Préparation des inocula}

A partir des pré-cultures de nuit, des colonies ont été prélevées et délayées dans $5 \mathrm{ml}$ de sérum physiologique. Les inocula de turbidité $0,3 \mathrm{McF}$ arland ont été préparés par mesure des densités optiques à une longueur d'onde de 625 $\mathrm{nm}$ à l'aide d'un spectrophotomètre de type UNICO comparable à une charge bactérienne de $1 \times 10^{8} \mathrm{UFC} / \mathrm{mL}$.

\section{Détermination des activités antimicrobiennes}

Les activités antimicrobiennes ont été évaluées par la méthode de diffusion dans la gélose. La gélose MH a été ensemencée par inondation avec $2 \mathrm{~mL}$ des inocula microbiens (bactériens et fongiques), puis les de boîtes Pétri ont été séchées pendant 15 minutes sous la hotte à flux laminaire à la température ambiante. Ensuite, les puits ont été réalisés dans la gélose (Alsarhan et al., 2013). Enfin, $50 \mu \mathrm{L}$ de chaque extrait d'une concentration de $100 \mathrm{mg} / \mathrm{mL}$ ont été déposés dans des puits de $6 \mathrm{~mm}$ de diamètres. Les boîtes ont été incubées à $37^{\circ} \mathrm{C}$ pendant $18 \mathrm{~h}$ à $24 \mathrm{~h}$.

Les activités antibactériennes et antifongiques ont été évaluées en mesurant, à l'aide d'un pied à coulisse analogique (Mitutoyo ABSOLUTE), le diamètre de la zone d'inhibition induite par les extraits, les antibiotiques et les antifongiques. Chaque test a été réalisé 3 fois.

\section{Détermination de la concentration minimale inhibitrice (CMI)}

La méthode de microdilution en milieu liquide a été utilisée pour déterminée les concentrations minimales inhibitrice (CMI) des extraits (Yala et al., 2017).

Globalement, une gamme de dilution géométrique de raison 2 des extraits a été réalisée avec des concentrations allant de 0,078 à $40 \mathrm{mg} / \mathrm{mL}$. Les témoins ont été préparés en additionnant $100 \mu \mathrm{L}$ du milieu avec de l'eau distillée stérile pour le témoin négatif, et la suspension bactérienne pour le témoin positif. Enfin, les microplaques ont été filmées et incubées à $37^{\circ} \mathrm{C}$ pendant $18 \mathrm{~h}$ à $24 \mathrm{~h}$.

\section{Détermination de la concentration minimale bactéricide (CMB)}

La concentration minimale bactéricide $(\mathrm{CMB})$ a été déterminée à partir de la concentration minimale inhibitrice (CMI). La CMB a été déterminée en ensemençant par étalement $100 \mu \mathrm{L}$ des échantillons des puits ne présentant aucune croissance visible à l'œil nu dans les microplaques après $18 \mathrm{~h}$ à $24 \mathrm{~h}$ d'incubation. La plus petite concentration qui ne laisse que $0,01 \%$ de bactéries survivantes après un temps d'exposition à l'extrait de $18 \mathrm{~h}$ à $24 \mathrm{~h}$ correspond à la CMB.

L'activité intrinsèque des extraits bruts nommé $\alpha$ a été déterminée en fonction du rapport CMB/CMI. Aussi, si $1 \leq \alpha$ $\leq 2$, l'effet est bactéricide et si $4 \leq \alpha \leq 16$, l'effet est bactériostatique. En outre, l'activité est dite tolérante pour toute valeur de $\alpha>16$.

\section{Analyse statistique}

Pour l'analyse statistique, le test Annova à un facteur a été utilisé pour comparer les diamètres d'inhibition observés sur les microorganismes. Le test de student a été employé pour comparer la variabilité des activités microbiennes (bactériennes et fongiques) selon les souches ou la plante. La réalisation de ces tests s'est effectuée à l'aide du logiciel R version 3.2.2 et le logiciel Excel (2013) a été utilisé pour le traitement de la base de données.

\section{Résultats:-}

\section{Screening phytochimique des plantes}

Le tableau 2 présente les grands groupes de familles chimiques contenus dans les deux extraits aqueux de plantes de $A$. floribunda et de B. ferruginea. Le screening phytochimique de ces extraits aqueux révèle la présence de plusieurs familles de composés chimiques. Ce sont, les alcaloïdes, les tannins, les polyphénols, les flavonoïdes, les stérols et 
les terpènes. La composition qualitative de ces métabolites dans les extraits aqueux de plantes montre des légères différences en fonction de la plante. Ainsi, nous notons une abondance des métabolites secondaires notamment les polyphénols, les tannins, les saponosides, les terpènes et stérols dans les deux espèces de plantes impliquées dans cette étude. Cependant, l'extrait aqueux de A. floribunda est très riches en saponosides tandis que ces métabolites sont à l'état de trace dans l'extrait B. ferruginea.

Les résultats soulignent également une présence de sucres réducteurs dans l'extrait aqueux de $B$. ferruginea contrairement à A. floribunda où ces composés sont absents.

Tableau 2:-Criblage phytochimique de A. floribunda et de B. ferruginea.

\begin{tabular}{|l|c|c|}
\hline \multirow{2}{*}{ Constituants chimiques } & \multicolumn{2}{|c|}{ Espèces } \\
\cline { 2 - 3 } & Alchornea floribunda & Bridelia ferruginea \\
\hline Tannins & +++ & ++ \\
Polyphénols & +++ & +++ \\
Flavonoïdes & +++ & +++ \\
Alcaloïdes & + & +++ \\
Stérols/terpènes & + & ++ \\
Hétérosides & ++ & ++ \\
Sucres réducteurs & - & ++ \\
Saponosides & +++ & + \\
Composés anthracéniques & ++ & +++ \\
\hline \multicolumn{2}{|r|}{+++ Très abondant } & ++ abondant \\
\hline
\end{tabular}

Potentiel antioxydant de $A$. floribunda et de B. ferruginea

L'activité antiradicalaire de $A$. floribunda et de $B$. ferruginea a été évaluée en référence à celles de l'acide gallique et de l'acide ascorbique. A l'analyse des résultats du tableau 3, les IC50 plus faibles sont obtenues avec les extraits aqueux de $A$. floribunda et de $B$. ferruginea, soient respectivement $18,8909 \pm 0,1045$ et $60,2797 \pm 0,0983 \mu \mathrm{g} \cdot \mathrm{mL}^{-1}$ Aussi, l'extraits aqueux de $A$. floribunda possède la plus forte activité antiradicalaire $\left(18,8909 \pm 0,1045 \mu \mathrm{g} \cdot \mathrm{mL}^{-1}\right)$ comparée à celle de B. ferruginea $\left(60,2798 \pm 0,0983 \mu \mathrm{g} \cdot \mathrm{mL}^{-1}\right)$.

Cependant, ces activités sont inférieures à celles de références respectivement de $1,5621 \pm 0,0335 \mu \mathrm{g} \cdot \mathrm{mL}^{-1}$ et 4,752 $\pm 0,0297 \mu \mathrm{g} \cdot \mathrm{mL}^{-1}$ pour l'acide ascorbique et l'acide gallique (Tableau 3).

Tableau 3:-Activité antioxydante de A. floribunda et de B. ferruginea

\begin{tabular}{|l|c|c|c|}
\hline \multicolumn{1}{|c|}{ Extraits/Standards } & Equations & $\mathbf{R}^{\mathbf{2}}$ & $\mathbf{I C}_{\mathbf{5 0}}\left(\boldsymbol{\mu g} \cdot \mathbf{m L}^{\mathbf{- 1}}\right)$ \\
\hline Acide ascorbique & $\mathrm{y}=1,112 \mathrm{x}+48,263$ & 0,3781 & $1,5621 \pm 0,0335$ \\
Acide gallique & $\mathrm{y}=1,1271 \mathrm{x}+44,644$ & 0,422 & $4,752 \pm 0,0297$ \\
A. floribunda & $\mathrm{y}=1,2804 \mathrm{x}+25,812$ & 0,7353 & $18,8909 \pm 0,1045$ \\
B. ferruginea & $\mathrm{y}=0,8897 \mathrm{x}-3,631$ & 0,8538 & $60,2798 \pm 0,0983$ \\
\hline
\end{tabular}

Pouvoir réducteur des plantes

La figure 2 montre les variations des absorbances qui traduisent le pouvoir réducteur, en fonction des concentrations des extraits des plantes étudiées et des substances standards.

L'extrait aqueux de A. floribunda présente un grand pouvoir réducteur du radical libre 2,2-diphényl-1picrylhydrazyle (DPPH) qui est supérieur à celui de $B$. ferruginea, et comparable à celui l'acide ascorbique et l'acide gallique aux concentrations élevées soient : 30,40 et $50 \mu \mathrm{g} \cdot \mathrm{mL}^{-1}$ car les absorbances des extraits aqueux de $A$. floribunda et de B. ferruginea sont faibles aux petites concentrations tandis qu'à de fortes concentrations, elles augmentent. 


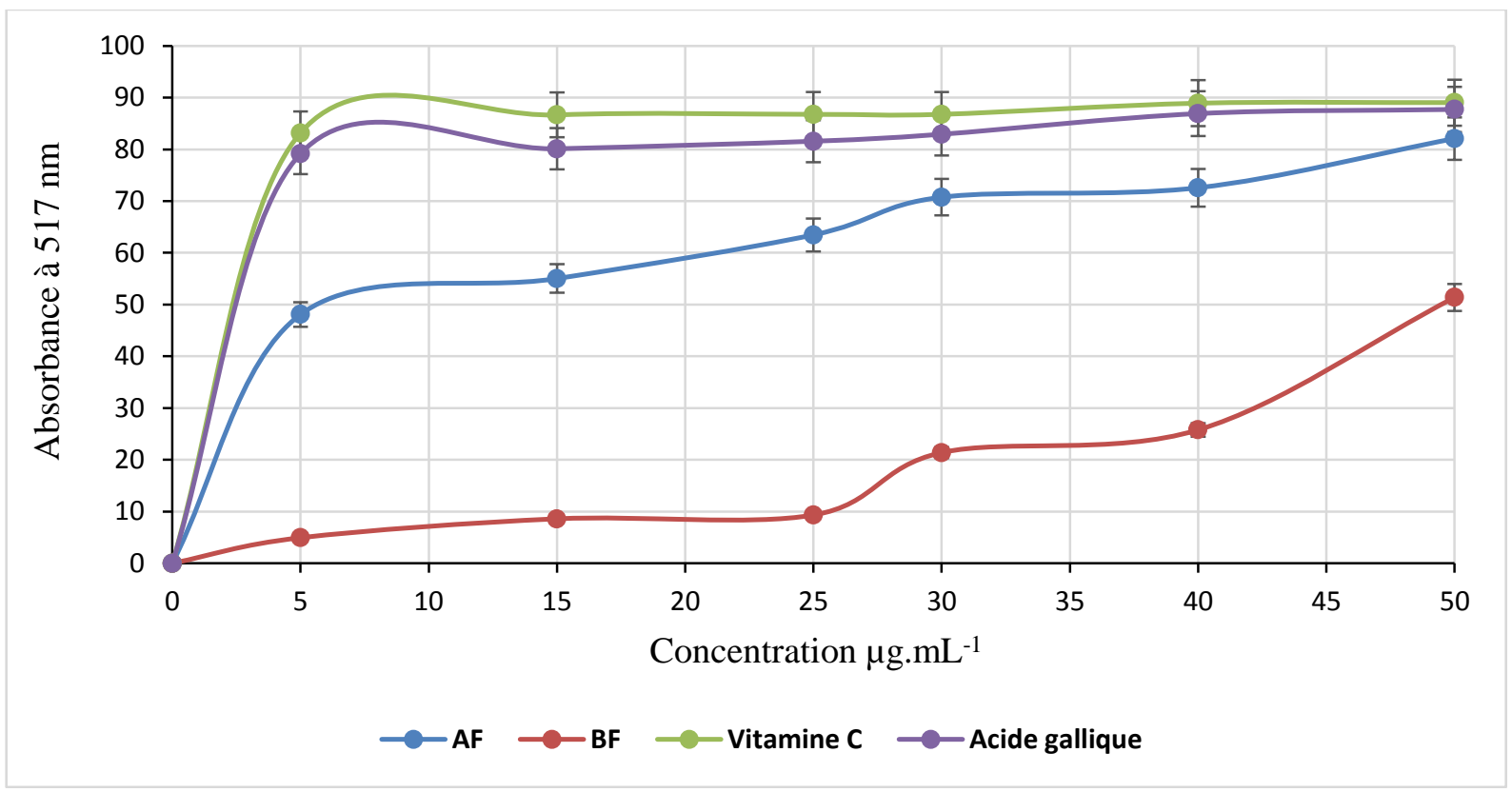

Figure 2:-Pouvoir réducteur des extraits et standards.

A.F : Alchornea floribunda ; BF : Bridelia ferruginea ;

\section{Indices d'activité antioxydant}

La figure 3 présente le résultat de l'indice d'activité antioxydante (IAA) des extraits aqueux de plantes et celles des références. Les résultats montrent que ces extraits ont les IAA les plus faibles soient $0,5293 \pm 0,1045$ pour $A$. floribunda et $0,165 \pm 0,0983$ pour $B$. ferruginea, comparativement à celles des molécules de références qui sont de $6,401 \pm 0,0335$ et 2,104 $\pm 0,0297$ respectivement pour l'acide ascorbique et l'acide gallique. Aussi, les extraits aqueux A. floribunda ont un meilleur indice d'activité antioxydante que celui de $B$. ferruginea avec 0,5293 $\pm 0,1045$ et $0,165 \pm 0,0983$ respectivement. Les résultats soulignent également que l'extrait de A. floribunda a une activité antioxydante modérée tandis que celui de $B$. ferruginea a une faible activité antioxydante. De plus, le test de student montre qu'il existe une différence significative sur la distribution moyenne de l'IAA selon les deux plantes étudiées $(\mathrm{p}=2.62 \cdot 10-7<0,05)$.

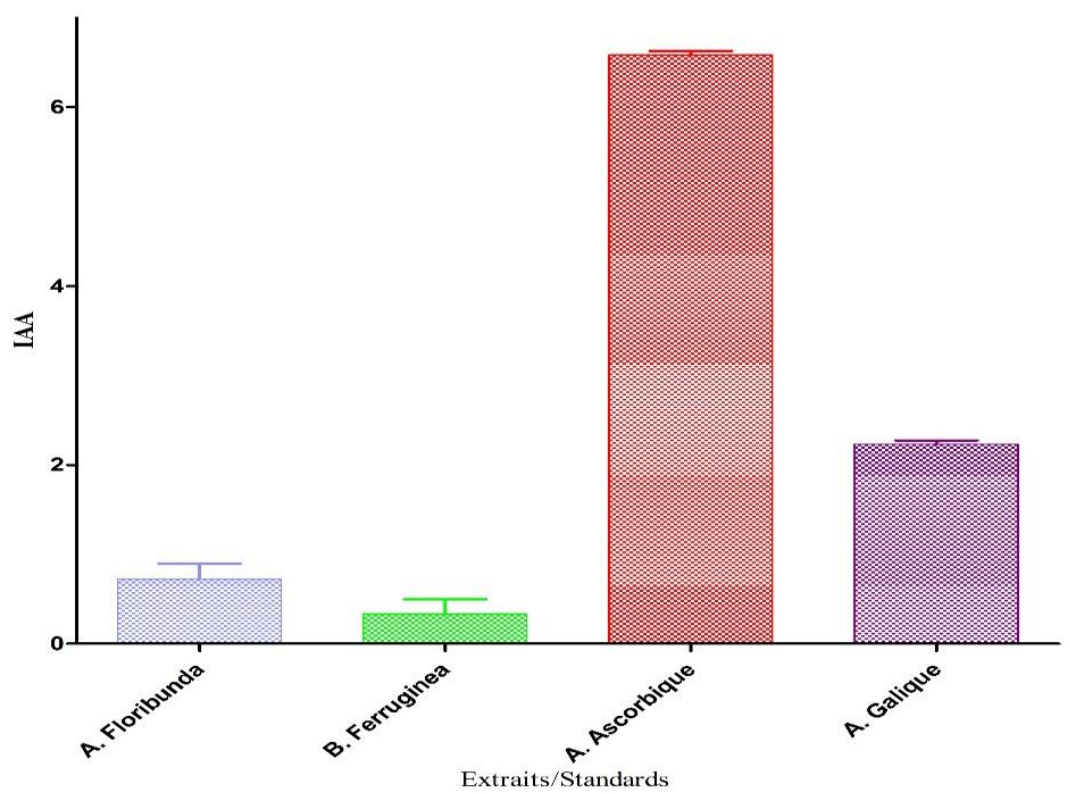

Figure 3:-Indices d'activité antioxydant des extraits et standards. 


\section{Activité antimicrobienne}

Dans cette étude, l'activité antimicrobienne a été évaluée en mesurant les diamètres d'inhibitions induits par les deux extraits aqueux de plantes à une concentration de $100 \mathrm{mg} \cdot \mathrm{mL}^{-1}$ sur les souches microbiennes testées. Les résultats obtenus sont présentés dans les tableaux 5 et 6 . Les valeurs consignées sont les moyennes de trois essais.

Test de sensibilité des bactéries aux extraits aqueux de A. floribunda et de B. ferruginea

Le tableau 4 présente les valeurs des différents diamètres d'inhibition $(\mathrm{mm})$ de la croissance des souches bactériennes.

Ces résultats révèlent que les deux extraits aqueux de plantes testés présentent une importante inhibition de la croissance de toutes les souches bactériennes. En effet, les diamètres d'inhibitions obtenus varient de 19,03 $\pm 2,52$ à $38,89 \pm 2,52 \mathrm{~mm}$ pour A. floribunda et de $20,4 \pm 2,52$ à $42,37 \pm 2,52 \mathrm{~mm}$ pour $B$. ferruginea. Les meilleures activités inhibitrices pour les deux extraits sont obtenues avec S. sonnei $(42,37 \pm 2,52)$, Shigella spp $(36,41 \pm 2,51)$, $R$. ornithinilytica $(26,83 \pm 2,89)$, $S$. choleraesuis $(23,68 \pm 2,52)$. De plus, les extraits aqueux de A. floribunda et de B. ferruginea présentent des zones d'inhibition supérieures ou égales à celles de la Gentamycine et le Chloramphénicol utilisé comme antibiotiques de référence dans cette étude (Tableau 4).

Toutefois, l'analyse de variance à un facteur montre qu'il existe une différence significative au seuil de $5 \%$ entre les diamètres d'inhibition des différentes bactéries étudiées $(\mathrm{p}=3,22 \cdot 10-19)$. Cependant, le test statistique de student montre qu'il n'existe aucune différence entre les diamètres d'inhibition des deux plantes $(p=0,33)$.

Tableau 4:-Diamètres des zones d'inhibition induites par les extraits aqueux de A. floribunda et de B. ferruginea.

\begin{tabular}{|c|c|c|c|c|c|c|c|c|c|c|}
\hline \multirow{2}{*}{$\begin{array}{c}\text { Extraits/ } \\
\text { Antibiotiqu } \\
\text { es }\end{array}$} & \multicolumn{10}{|c|}{$\mathrm{CMB} / \mathrm{CMI}(\mathrm{mg} / \mathrm{mL})$} \\
\hline & $\begin{array}{c}S . \\
\text { cholera } \\
\text { esuis }\end{array}$ & $\begin{array}{c}S . \\
\text { parathy } \\
\text { phie }\end{array}$ & $\begin{array}{c}\text { Se. } \\
\text { ordorif } \\
\text { era }\end{array}$ & $\begin{array}{c}K . \\
\text { oxytoc } \\
a\end{array}$ & $\begin{array}{c}S . \\
\text { sonnei }\end{array}$ & $\begin{array}{c}R . \\
\text { ornithini } \\
\text { lytica }\end{array}$ & $\begin{array}{l}\text { Shigel } \\
\text { la spp }\end{array}$ & $\begin{array}{c}Y . \\
\text { pestis }\end{array}$ & $\begin{array}{c}E . \\
\text { vulner } \\
\text { is }\end{array}$ & $\begin{array}{c}E . \\
\text { colis }\end{array}$ \\
\hline $\begin{array}{l}\text { A. } \\
\text { floribunda }\end{array}$ & $\begin{array}{c}23,68 \pm \\
2,52\end{array}$ & $\begin{array}{c}20,2 \pm \\
2,52\end{array}$ & $\begin{array}{c}19,03 \pm \\
2,52\end{array}$ & $\begin{array}{r}24,27 \\
\pm 2,52\end{array}$ & $\begin{array}{r}38,89 \\
\pm 2,52\end{array}$ & $\begin{array}{c}26,83 \pm \\
2,89\end{array}$ & $\begin{array}{r}36,41 \\
\pm 2,51\end{array}$ & $\begin{array}{l}27,348 \\
\pm 2,52\end{array}$ & $\begin{array}{r}24,46 \\
\pm 2,52\end{array}$ & $\begin{array}{r}21,47 \\
\pm 2,52\end{array}$ \\
\hline $\begin{array}{l}\text { B. } \\
\text { ferruginea }\end{array}$ & $\begin{array}{c}23,68 \pm \\
2,52 \\
\end{array}$ & $\begin{array}{c}23,92 \pm \\
2,08\end{array}$ & $\begin{array}{c}20,4 \pm \\
2,52 \\
\end{array}$ & $\begin{array}{c}24,9 \pm \\
2,52 \\
\end{array}$ & $\begin{array}{r}42,37 \\
\pm 2,52 \\
\end{array}$ & $\begin{array}{c}25,74 \pm \\
2,52 \\
\end{array}$ & $\begin{array}{r}36,23 \\
\pm 2,52 \\
\end{array}$ & $\begin{array}{r}23,62 \\
\pm 2,52 \\
\end{array}$ & $\begin{array}{r}24,02 \\
\pm 2,52 \\
\end{array}$ & $\begin{array}{r}25,64 \\
\pm 2,52 \\
\end{array}$ \\
\hline $\begin{array}{l}\text { Gentamycin } \\
\text { e }\end{array}$ & $\begin{array}{c}26,3 \pm \\
1,15\end{array}$ & $\begin{array}{c}25,67 \pm \\
1\end{array}$ & $\begin{array}{c}29,43 \pm \\
1\end{array}$ & $\begin{array}{c}26,65 \\
\pm 1\end{array}$ & $\begin{array}{c}25,34 \\
\pm 1\end{array}$ & $9,98 \pm 1$ & $\begin{array}{c}23,34 \\
\pm 1\end{array}$ & $\begin{array}{c}27,88 \\
\pm 1\end{array}$ & $\begin{array}{c}26,05 \\
\pm 1\end{array}$ & $\begin{array}{c}25,47 \\
\pm 1\end{array}$ \\
\hline $\begin{array}{c}\text { Chloramph } \\
\text { énicol }\end{array}$ & $9,3 \pm 1$ & $8,5 \pm 1$ & $34 \pm 1$ & $\begin{array}{c}32,36 \\
\pm 1 \\
\end{array}$ & $\begin{array}{c}31,4 \pm \\
1 \\
\end{array}$ & $32,1 \pm 1$ & $\begin{array}{c}16,86 \\
\pm 1 \\
\end{array}$ & $\begin{array}{c}31,04 \\
\pm 1 \\
\end{array}$ & $\begin{array}{c}29,85 \\
\pm 1 \\
\end{array}$ & $\begin{array}{r}32,05 \\
\pm 0,98\end{array}$ \\
\hline
\end{tabular}

S. choleraesuis: Salmonella choleraesuis ; S. parathyphie: Salmonella parathyphie ; Se ordorifera: Serratia ordorifera ; K. oxytoca: Klebsiella oxytoca ; R. ornithinilytica: Raoultella ornithinilytica ; S. sonnei: Shigella sonnei; Y. pestis: Yersinia pestis ;E. vulneris: Escherichia vulneris ; E. coli: Escherichia coli.

Test de sensibilité des levures aux extraits aqueux de A. floribunda et de B. ferruginea

Le tableau 5 présente les valeurs des différents diamètres d'inhibition $(\mathrm{mm})$ de la croissance des souches fongiques. Ces résultats soulignent que les extraits aqueux des plantes testés présentent des diamètres d'inhibition moyens sur la croissance de toutes les souches fongiques, car les diamètres obtenus varient de $11,1 \pm 2,02$ à 13,04 $\pm 2,7 \mathrm{~mm}$ pour A. floribunda et de 12,03 $\pm 1,79$ à 15,85 $\pm 3,07$ pour B. ferruginea. Les extraits aqueux de A. floribunda et B. ferruginea présentent des zones d'inhibition supérieures à celles de la Nystatine et l'Amphotéricine B sur toutes les souches fongiques exceptée la souche de Candida albicans d'origine buccale. En outre, les extraits aqueux de $B$. ferruginea montrent des meilleures zones d'inhibition que A. floribunda sur toutes les souches fongiques (Tableau $5)$.

Le test de variance Annova à un facteur montre qu'il n'existe aucune différence significative entre les quatre souches fongiques étudiées et les diamètres d'inhibition observés, au seuil de significativité de $5 \%(\mathrm{p}=0,37)$. Le test de student montre qu'il n'existe aucune différence entre les diamètres d'inhibition des deux plantes étudiées ( $\mathrm{p}=$ 0,07). Par ailleurs, le test de parité de TUCKEY montre qu'il n'existe toujours aucune différence significative si l'on compare les souches fongiques deux par deux $(\mathrm{p}>0,05)$. 
Tableau 5:-Diamètres des zones d'inhibitions induites par les extraits aqueux de A. floribunda et de $B$. ferruginea

\begin{tabular}{|l|c|c|c|c|}
\hline \multirow{2}{*}{ Extraits / Antifongiques } & \multicolumn{4}{|c|}{ Diamètres d'inhibition (mm) } \\
\cline { 2 - 5 } & Candida spp PV & Candida albicans PV & Candida albicans bucal & Candida spp PV \\
\hline A. floribunda & $\mathbf{1 1 , 9 3} \pm \mathbf{0 , 2 4}$ & $\mathbf{1 1 , 5 9} \pm \mathbf{2 , 9 6}$ & $\mathbf{1 1 , 1} \pm \mathbf{2 , 0 8}$ & $\mathbf{1 3 , 0 4} \pm \mathbf{2 , 7}$ \\
\hline B. ferruginea & $\mathbf{1 5 , 8 5} \pm \mathbf{3 , 0 7}$ & $\mathbf{1 2 , 0 3} \pm \mathbf{1 , 7 9}$ & $\mathbf{1 3 , 6 2} \pm \mathbf{1 , 3}$ & $14,32 \pm 2$ \\
\hline Amphotéricine B & $\mathbf{0}$ & $\mathbf{0}$ & $\mathbf{1 3 , 9 2} \pm \mathbf{1}$ & $11,4 \pm \mathbf{1}$ \\
\hline Nystatine & $\mathbf{9 , 6 5} \pm \mathbf{1}$ & $\mathbf{1 0 , 3 5} \pm \mathbf{1}$ & $\mathbf{1 4 , 5 5} \pm \mathbf{1}$ & $13,5 \pm 1$ \\
\hline
\end{tabular}

PV : Prélèvement vaginal

Concentrations minimales inhibitrices (CMI) de A. floribunda et de B. ferruginea

Les différentes concentrations minimales inhibitrices (CMI) obtenues pour chaque extrait végétal testé sont présentées dans le tableau 6. Les valeurs consignées sont les moyennes de trois essais. Chaque extrait a des activités variables (pouvoir inhibiteur) qui sont étroitement liées à leur composition en métabolites secondaires et de la souche bactérienne en présence.

Ces résultats révèlent que les deux extraits aqueux de plantes ont des activités inhibitrices sur toutes les souches testées avec des CMI variant entre $0,117 \pm 0,039$ et 5,625 $\pm 4,375 \mathrm{mg}$. $\mathrm{mL}^{-1}$. Les extraits aqueux de A. floribunda et de $B$. ferruginea ont des activités antibactériennes à des faibles concentrations minimales inhibitrices $(0,117 ; 0,312$; 0,468 et $0,937 \mathrm{mg}$. $\mathrm{mL}^{-1}$ ) sur plusieurs souches à savoir : Salmonella parathyphie, Klebsiella oxytoca, Raoultella ornithinilytica, Shigella_sonnei, Yersinia pestis et Escherichia coli.

Tableau 6:-Concentration minimale inhibitrice (CMI) des extraits.

\begin{tabular}{|c|c|c|c|c|c|c|c|c|c|c|}
\hline \multirow{3}{*}{$\begin{array}{l}\text { Espèces } \\
\text { végétales }\end{array}$} & \multicolumn{10}{|c|}{ CMB/CMI (mg.mL ${ }^{-1}$ ) } \\
\hline & & & & & Iicroorgan & & & & & \\
\hline & SC & SPA & SO & KO & SHS & RO & SHP & YP & $\mathbf{E V}$ & EC \\
\hline A. floribunda & $\begin{array}{c}5,312 \\
\pm 4,687\end{array}$ & $\begin{array}{c}0,937 \pm 0,3 \\
12\end{array}$ & $\begin{array}{c}5,625 \pm 4,3 \\
75\end{array}$ & $\begin{array}{c}0,312 \pm \\
0\end{array}$ & IND* & $\begin{array}{c}0,312 \pm \\
0\end{array}$ & $\begin{array}{c}0,468 \pm 0,1 \\
56\end{array}$ & $\begin{array}{c}0,117 \pm 0,0 \\
39\end{array}$ & $\begin{array}{c}\text { IND } \\
*\end{array}$ & $\begin{array}{c}0,312 \pm \\
0\end{array}$ \\
\hline B. ferruginea & $\begin{array}{c}5,312 \\
\pm 4,687\end{array}$ & $\begin{array}{c}0,937 \pm 0,3 \\
12\end{array}$ & $\begin{array}{c}5,625 \pm 4,3 \\
75\end{array}$ & IND* & $\begin{array}{c}0,937 \pm 0,3 \\
12\end{array}$ & $\begin{array}{c}0,312 \pm \\
0\end{array}$ & $\begin{array}{c}0,468 \pm 0,1 \\
56\end{array}$ & $\begin{array}{c}0,117 \pm 0,0 \\
39\end{array}$ & $\begin{array}{c}\text { IND } \\
*\end{array}$ & $\begin{array}{c}0,312 \pm \\
0\end{array}$ \\
\hline
\end{tabular}

SC: Salmonella choleraesuis ; SPA: Salmonella parathyphie ; SO: Serratia ordorifera ; KO: Klebsiella oxytoca ; RO: Raoultella ornithinilytica ; SHS: Shigella sonnei; SHP : Shigella sp ;YP: Yersinia pestis ;EV: Escherichia vulneris ; EC: Escherichia coli. IND* : CMI>40 mg/Ml

\section{Concentrations minimales bactéricides (CMB)}

Les résultats du tableau 7 présentent les valeurs de la concentration minimale bactéricide des extraits aqueux de plantes sur les dix souches bactériennes. Il ressort que les concentrations minimales bactéricides sont obtenues à des faibles doses soient : $0,234 \pm 0,078 ; 0,625 ; 0,937 \pm 0,312 ; 1,25$ et 1,875 $\pm 0,625 \mathrm{mg} \cdot \mathrm{mL}^{-1}$. Ces résultats sont obtenus sur cinq (5) souches à savoir: Klebsiella oxytoca, Raoultella ornithinilytica, Salmonella parathyphie, Shigella sonnei, Yersinia pestis et Escherichia coli.

Tableau 7:-Concentration minimale bactéricide (CMB) des extraits.

\begin{tabular}{|c|c|c|c|c|c|c|c|c|c|c|}
\hline \multirow{3}{*}{$\begin{array}{c}\text { Espèces } \\
\text { végétales }\end{array}$} & \multicolumn{10}{|c|}{ CMB/CMI (mg.mL ${ }^{-1}$ ) } \\
\hline & \multicolumn{10}{|c|}{ Microorganismes } \\
\hline & SC & SPA & SO & KO & SHS & RO & SHP & YP & EV & EC \\
\hline A. floribunda & $\begin{array}{c}20,625 \\
\pm 19,375\end{array}$ & $\begin{array}{c}1,25 \\
\pm 0\end{array}$ & $\begin{array}{c}11,25 \pm 8 \\
75\end{array}$ & $\begin{array}{c}0,625 \\
\pm 0\end{array}$ & IND* & $\begin{array}{c}0,937 \pm 0 \\
312\end{array}$ & $\begin{array}{c}0,625 \\
\pm 0\end{array}$ & $\begin{array}{c}0,234 \pm 0 \\
078\end{array}$ & $\begin{array}{c}\text { IND } \\
*\end{array}$ & $\begin{array}{c}0,937 \pm 0 \\
312\end{array}$ \\
\hline B. ferruginea & $\begin{array}{c}20,625 \\
\pm 19,375 \\
\end{array}$ & $\begin{array}{c}1,25 \\
\pm 0 \\
\end{array}$ & $\begin{array}{c}11,25 \pm 8 \\
75\end{array}$ & IND* & $\begin{array}{c}1,875 \pm 0 \\
625\end{array}$ & $0,625 \pm 0$ & $\begin{array}{c}0,625 \\
\pm 0 \\
\end{array}$ & $\begin{array}{c}0,234 \pm 0 \\
078\end{array}$ & $\begin{array}{c}\text { IND } \\
*\end{array}$ & IND* \\
\hline
\end{tabular}

SC: Salmonella choleraesuis ; SPA: Salmonella parathyphie ; SO: Serratia ordorifera ; KO: Klebsiella oxytoca ; RO: Raoultella ornithinilytica ; SHS: Shigella sonnei ; SHP : Shigella sp ;YP: Yersinia pestis ;EV: Escherichia vulneris ; EC: Escherichia coli.

\section{Rapports CMB/CMI des extraits aqueux de A. floribunda et de B. ferruginea}

Les résultats du tableau 8 font état des différents rapports CMB/CMI de A. flribunda et B. ferruginea. Ces différents rapports obtenus varient de 1,334 à 3,882 pour les deux extraits de plantes. De manière générale, les deux extraits aqueux ont une activité bactéricide vis-à-vis des souches notamment Salmonella parathyphie, Serratia ordorifera, Shigella sonnei, Klebsiella oxytoca, Shigella sp, Yersinia pestis, Raoultella ornithinilytica. 
Tableau 8:-Rapport CMB/CMI des extraits aqueux de A. florbunda et B. ferruginea

\begin{tabular}{|c|c|c|c|c|c|c|c|c|c|c|}
\hline \multirow[t]{3}{*}{ Espèces végétales } & \multicolumn{10}{|c|}{ CMB/CMI (mg/mL) } \\
\hline & \multicolumn{10}{|c|}{ Microorganismes } \\
\hline & SC & SPA & SO & KO & SHS & RO & SHP & YP & EV & EC \\
\hline A. floribunda & 3,882 & 1,334 & 2 & 2,003 & IND* & 3,003 & 1,335 & 2 & IND* & 3,003 \\
\hline B. ferruginea & 3,882 & 1,334 & 2 & IND* & 2,001 & 2,003 & 1,335 & 2 & IND* & IND* \\
\hline
\end{tabular}

SC: Salmonella choleraesuis ; SPA: Salmonella parathyphie ; SO: Serratia ordorifera ; KO: Klebsiella oxytoca ; RO: Raoultella ornithinilytica ; SHS: Shigella sonnei ; SHP : Shigella sp ;YP: Yersinia pestis ;EV: Escherichia vulneris ; EC: Escherichia coli. ; IND* : CMB $>40$ mg.mL ${ }^{-1}$

\section{Discussion:-}

Le but de ce travail était d'évaluer les activités biologiques des extraits aqueux de deux plantes (A. floribunda et $B$. ferruginea).

Les résultats du screening phytochimique ont révélé une abondance de tannins, de stérols, des saponosides, des terpènoïdes, des polyphénols, des flavonoïdes, des composés anthracéniques, des anthocyanes, des hétérosides et des alcaloïdes dans les plantes (tableau 2). Ces molécules sont des métabolites secondaires qui confèrent aux plantes leurs activités antibactériennes, antifongiques, antiparasitaires, antivirales et antioxydantes (Merghache, Boucherit, and Boucherit, 2012). Les résultats obtenus sont similaires à ceux de Nsi Akoué et al. (Nsi AKoué et al., 2013) qui ont montré que Agelaea pentagyna et Dialium dinklagei, plantes consommées par les mandrills (Mandrillus sphinx) du parc de la Lekédi ont une richesse en métabolites secondaires notamment les flavonols, les flavones, les triterpènes et les coumarines intervenant dans le stress oxydatif.

Le stress oxydatif est directement ou indirectement responsable de nombreuses maladies qui affectent fortement la santé humaine. La présence en excès des espèces réactives de l'oxygène (ERO) consécutive au métabolisme aérobie chez les mammifères, provoqué par un déséquilibre dans l'organisme, entre les processus d'oxydation et de défense antioxydante (Eyi Mintsa, 2017), engendre le stress oxydatif qui est une des causes majeures des affections telles que les maladies métaboliques, les cancers, les maladies neurodégénératives, les processus de vieillissement tissulaires (Bidié et al., 2008). Un apport en molécules bioactives à effets antioxydants par la consommation des fruits et légumes et le recours aux plantes, devient une alternative pour pallier au déficit des mesures endogènes de défense antioxydante (Cardona et al., 2013; Lahouel et al., 2004; Pincemail et al., 2007; Shoji et al., 2004).

Les résultats issus de l'évaluation de l'activité antioxydante et antiradicalaire montrent que l'extrait aqueux de A. floribunda a une activité antioxydante modérée tandis que celui de $\mathrm{B}$. ferruginea est faible en référence à l'échelle d'évaluation de l'activité antioxydante de Scherer and Godoy (2009). De plus, le test de student montre qu'il existe une différence significative sur la distribution moyenne de l'IAA selon les deux plantes étudiées $(\mathrm{p}=2.62 .10$ $7<0,05)$.

En effet, la capacité d'une molécule à piéger les radicaux libres est liée à ces composés chimiques qui auraient la capacité de céder facilement un électron ou un proton. Les études de Zongo et al. (Zongo et al., 2010) ont montré que les composés phénoliques ont une relative facilitée à céder un électron ou un proton pour piéger les radicaux libres comme les ERO.

Les plantes qui font l'objet de cette étude pourraient être introduites comme complément alimentaire dans l'alimentation humaine, car les molécules à pouvoir antioxydant sont fortement recommandées dans la mesure où elles renforceraient le système de défense antioxydant et permettraient ainsi de lutter contre les affections liées à l'âge et aux désordres métaboliques comme le cancer, les maladies cardiovasculaires et les maladies neurodégénératives (Hsu, Chan, and Chang, 2007; Li et al., 2008).

Par ailleurs, les résultats de l'activité antibactérienne et antifongique des extraits aqueux de A. floribunda et de B. ferruginea (tableaux 5 et 6) montrent que ces plantes inhibent le développement de toutes les souches microbiennes testées.

La forte inhibition de la croissance des bactériens responsables des diarrhées pourrait s'expliquer par les résultats du criblage phytochimique des plantes (tableau 2), qui révèle la présence abondante des métabolites secondaires tels que : les tannins, les alcaloïdes, les flavonoïdes, les composés terpéniques et les polyphénols dans les deux extraits analysés (Merghache, Boucherit, and Boucherit, 2012). Assurément, les composés tels que les alcaloïdes, les 
flavonoïdes, les polyphénols, les tannins, les stérols, les anthraquinones sont dotés des propriétés antibactériennes et antifongiques (Moroh et al., 2008; Tene et al, 2009). Les différentes classes de flavonoïdes (flavonols, flavones) et des tannins (gallique, catéchine) isolées au niveau des feuilles et des écorces de A. floribunda ont montré une activité antibactérienne in vivo contre Staphylococus aureus et Escherichia coli (Okoye et al., 2014). Aussi, cette activité trouverait son origine par le mécanisme d'action des phytomolécules qui agiraient probablement par destruction des membranes externes et internes des bactéries (Falconer and Brown, 2009). Elles peuvent également agir par d'autres mécanismes car il a été démontré que les métabolites secondaires impliqueraient divers mécanismes d'action qui diffèreraient de ceux des molécules d'antibiotiques conventionnelles (Falconer and Brown, 2009; Tene et al., 2009; Yala et al., 2017).

De même, les résultats montrent également que les extraits aqueux de A. floribunda et de B. ferruginea ont des activités antifongiques sur toutes les souches de Candida. Ces résultats vont dans le même sens que ceux de Batawila (Batawila, 2002), qui a montré que les extraits aqueux d'écorces de tronc de Pteleopsis. suberosa inhibent significativement $C$. albicans, Microsporum gypseum, Epidermophyton floccosum, Trichophyton mentagrophytes et Tricophyton rubrum. L'inhibition de la croissance pourrait se traduire par la présence des composés chimiques tels que : les tannins, les terpènes, les flavonoïdes et les polyphénols (tableau 2). En effet, les terpènoïdes, les tannins sont connus comme des molécules douées de propriétés antifongiques (Merghache, Boucherit, and Boucherit, 2012). Les travaux de Ochi et al. (Ochi et al., 2015) ont montré que les tannins catéchiques et les tannins galliques présents dans les feuilles et les racines de A. floribunda avaient des activités antifongiques notamment sur Candida albicans. De plus, Kporou Kouassi et al. (Kporou Kouassi et al., 2009) ont révélé que l'extrait possédant uniquement les stérols et les triterpènes présentait une meilleure activité antifongique. Candida albicans est le principal agent pathogène des candidose buccales et vaginales, l'usage naturelle de ces plantes comme agents alternatifs pour le contrôle des infections fongiques serait une alternative intéressante (Kamurthy et al., 2016).

Cependant, l'activité antifongique obtenue dans cette étude est moins importante que celle des bactéries. Cette différence pourrait probablement être due à la différence des mécanismes d'action des phytomolécules sur les deux types de microorganismes qui ont fait l'objet de cette étude. Effectivement, les diamètres d'inhibitions moyens observés sur les levures pourraient s'expliquer par la faible concentration cytoplasmique ou la faible affinité des phytomolécules pour leur cible dans le cytoplasme des souches de Candida (Guillot and Dannaoui, 2015). Aussi, la facilité avec laquelle Candida albicans peut changer de phénotype serait en relation avec sa résistance aux antifongiques (Yang et al., 2005) et probablement la diminution de l'activité antifongiques de ces extraits. Ce mécanisme serait la source de l'émergence des souches $C$. albicans résistantes aux antifongiques usuels et rend difficiles leurs éradications.

Enfin, les résultats de l'activité antibactérienne des extraits aqueux de A. floribunda et $B$. ferruginea ont permis de déterminer la CMI, la $\mathrm{CMB}$ et le rapport $\alpha \mathrm{CMB} / \mathrm{CMI}$, qui a permis de donner le profil de sensibilité des extraits visà-vis des souches bactériennes. Les faibles concentrations minimales inhibitrices et bactéricides suggèrent que les extraits aqueux de A. floribunda et de B. ferruginea ont une activité bactéricide sur toutes les souches bactériennes testées.

Bien que ces souches microbiennes soient reconnues comme étant résistantes à plusieurs antibiotiques et antifongiques, les résultats de l'activité antibactérienne et antifongique suggèrent que les extraits aqueux de $A$. floribunda et de $B$. ferruginea constitueraient un moyen préventif ou de lutte contre les affections microbiennes. Ces résultats justifient les usages pharmacologiques des plantes $A$. floribunda et de $B$. ferruginea par les populations indigènes en médecine traditionnelle à des fins thérapeutiques comme antibactériens, antifongiques et antiparasitaires (Musuyu Muganza et al., 2012; Ondo, 2011).

\section{Conclusion:-}

Cette étude avait pour objectif d'évaluer les activités biologiques et pharmacologiques de $A$. floribunda et $B$. ferruginea.

Le criblage phytochimique de ces extraits aqueux de plantes a montré une abondance en métabolites secondaires : les tanins, les saponosides, les flavonoïdes, les alcaloïdes, les polyphénols et les stérols qui ont des propriétés pharmacologiques (antibactériennes, antifongiques, antioxydantes, antivirales et antiparasitaires). Elles possèdent également un potentiel antioxydant modéré pour A. floribunda et faible pouvoir antioxydant pour B. ferruginea. 
Les résultats des activités antimicrobiennes ont révélé un important pouvoir antimicrobien sur toutes les souches testées. Une importante activité antibactérienne a été trouvée sur toutes les souches bactériennes isolées des diarrhées infantiles. Les souches les plus sensibles étant $S$. sonnei et Shigella spp qui sont des enteropathogènes diarrhéiques. Une remarquable activité antifongique a été observée vis-à-vis des champignons, mais les plus sensibles étaient Candida albicans bucal et Candida spp PV.

Ainsi, ces plantes pourraient être une nouvelle source de substances dotées d'activités biologiques pour des usages thérapeutiques afin de pallier au phénomène de résistance que pose certaines pathologies, tant sur le plan métabolique, parasitaire que microbien.

Bien qu'encourageant, nos résultats restent préliminaires et nécessitent d'être approfondies, notamment pour déterminer :

1. Le potentiel nutritif de ces plantes ;

2. Les mécanismes d'action des différentes molécules contenues dans ces extraits ;

3. La toxicité de ces deux plantes.

\section{Références Bibliographiques:-}

1. Alsarhan, A., Mansi, K., Aburjai, T., Al-khatib, A., Alzweiri, M., and Sultana, N. (2013). HSP70 and inos biomarkers in evaluating the healing properties of rubia tinctorum. European Scientific Journal, 9(15), 241-254.

2. Batawila, K. (2002). Diversité, écologie et propriétés antifongiques des Combretaceaea du Togo. Acta Bot. Gallica. Univ. Reims et Univ Lomé.

3. Bidié, A. P., Koffi, E., N'guessan, J. D., Djaman, A. J., and Guédé-Guina, F. (2008). Influence of Mitragyna ciliata (Myta) on the microsomalactivity of ATPase $\mathrm{Na}+/ \mathrm{K}+$ dependent extract on a rabbit (heart) African. Int. J. Biomol. Biomed., 5, 294-301.

4. Bouquet, A., and Fouret, A. (1975). Recherches chimiques préliminaires sur les plantes médicinales du CongoBrazzaville. Fitoterapia, 4, 175-177.

5. Cardona, F., Andrés-lacueva, C., Tulipani, S., Tinahones, F., and Queipo-ortuño, M. (2013). Benefits of polyphenols on gut microbiota and implications in human health. The Journal of Nutritional Biochemistry, 24(8), 1415-1422. https://doi.org/10.1016/j.jnutbio.2013.05.001

6. Eyi Mintsa, A. B. (2017). Etude des propriétés pharmacologiques et physiologique de Helichrysum mechowianum (Asteriaceae), plante utilisée par les pygmées du Gabon. Thèse. Université des Sciences et Techniques de Masuku (Gabon).

7. Falconer, S. B., and Brown, E. D. (2009). New screens and targets in antibacterial drug discovery. Current Opinion in Microbiology, 12(5), 497-504.

8. Gruber, J., Schaffer, S., and Barry, H. (2008). The mitochondrial free radical theory of ageing - Where do we stand? Frontiers in Bioscience, 13, 6554-6579.

9. Guillot, J., and Dannaoui, E. (2015). La résistance aux antifongiques: importance en médecine humaine et véritérinaire. Bull. Acad. Vét. France, Tome 168(4), 314-319.

10. Hsu, C. Y., Chan, Y. P., and Chang, J. (2007). Antioxidant activity of extract from Polygonum. Biol Res, 40, $13-21$.

11. Huffman, M. A. (2003). Animal self-medication and ethno-medecine: exploration and exploitation of the medication properties of plants. Proceeding of the Nutr Soc, 62, 371-381.

12. Kamurthy, H., Tejmal, M., Majumder, P., and Ambujakshi, H. R. (2016). Antifungal Activity of Weed Extracts on Candida albicans. An In-vitro Study. International Journal of Phytomedicine, 8, 453-456. https://doi.org/10.5138/09750185.1847

13. Konaté, K., Souza, A., Kassi Yomalan, T., Dibala, I. C., and Barro, N. (2011). Phytochemical composition, Antioxidant and Anti-inflammatory potential of bioactive fractions from extracts of three medicinal plants traditionally used to treat liver diseases in Burkina Faso. International Journal of Phytomedicine, 3, 406-415.

14. Kporou Kouassi, E., Kra Adou Koffi, M., OUattara, S., and Guede-Guina, F. (2009). Évaluation de la sensibilité de Candida albicans aux extraits de Mitracarpus scaber une Rubiaceae codifiée Misca. Bulletin de La Société Royale Des Sciences de Liège, 78, 12-23.

15. Lahouel, M., Boulkour, S., Segueni, N., and Fillastre, J. P. (2004). du cyclophosphamide et du paracétamol par inhibition de la peroxydation lipidique et augmentation du glutathion hépatique The flavonoids effect against vinblastine, cyclophosphamide and paracetamol toxicity by inhibition of lipid-peroxydation and increas. Pathologie Biologie, 52, 314-322. https://doi.org/10.1016/j.patbio.2004.01.001 
16. Devers, D., Vande weghe, J.P., Billand, A., Cassagne, B., Doucet, J.L., Nasi, R., Tréfon, T., and Tutin, C. (2006). Les forêts du Bassin du Congo : Etat des Forêts 2006.

17. Li, H., Wang, X., Li, P., Li, Y., and Wang, H. (2008). Comparative Study of Antioxidant Activity of Grape (Vitis vinifera ) Seed Powder Assessed by Different Methods. Journal of Food and Drug Analysis, 16(6), 1-8.

18. Mengome. (2013). Structures-Activités biologiques des polysaccharides de quelques plantes endémiques du Gabon. Thèse, $243 \mathrm{p}$.

19. Merghache, D., Boucherit, A. Z., and Boucherit, K. (2012). Évaluation de l'activité antifongique de différents extraits de la cannelle de Chine (Cinnamomum cassia). Phytothérapie, 10(4), 215-221.

20. Merghache, D., Boucherit, A. Z., and Boucherit, K. (2012). Evaluation de l'activité antifongique des différents extraits de la cannelle de Chine (Cinnamomum cassia). Phyto, 10(4), 215-221.

21. Moroh, J. A., Bahi, C., Dje, K., and Loukou, Y. G. (2008). Étude de l' activité antibactérienne de 1 ' extrait acétatique (EAC) de Morinda morindoides (Baker) milne-redheat (rubiaceae) sur la croissance in-vitro des souches d'Escherichia coli Study of the antibacterial activity of Morinda morindoides (Baker). Bulletin de La Société Royale Des Sciences de Liège, 77, 44-61.

22. Musuyu Muganza, D., Fruth, B. I., Nzunzu Lami, J., Mesia, G., Kambu, O. K., Tona, G., and Pieters, L. (2012). In vitro antiprotozoal and cytotoxic activity of 33 ethonopharmacologically selected medicinal plants from Democratic Republic of Congo. J Ethnopharmacol., 141(1), 301-8.

23. Nsi Akoué, G. (2017). Etude des plantes médicinales et/ou alimentaires issues du régime alimentaire du mandrill (Mandrillus sphinx) : Approche Zoopharmacognosie. Thèse. Université Des Sciences et Techniques de Masuku (Gabon), 176 pages.

24. Nsi AKoué, G., Obame, L. C., ONDO, J., Brama, I., Mbadinga-Mbading, W., Otogo N'nang, E. S., and Mbatchi, B. (2013). phytochemical and antiradical activities of Agelaea pentagyna (Lam) Baill and Dialium dinklagei Harms. Medicinal plants from Gabon. International Journal of Advanced Research, 1(8), $246-255$.

25. Nsi Akoué, G., Obame, L. C., Ondo, J. P., Brama, I., Otogo N’Nang, E. S., Tapoyo, S. Y., and Souza, A. (2013). Phytochemical composition and antiradical activity of Sakersia africana Hook. f. medicinal plant from Gabon Sakersia total. Int. J. Biomol. Biomed. Introduction, 3(3), 1-8.

26. Ochi, I. O., Ogah, E., Longbap, B. D., Abiaziem, C. V., and Tabe, N. T. (2015). PHYTOCHEMICAL ANALYSIS AND ANTIMICROBIAL SCREENING OF DRIED ROOT. Ewemen Journal of Microbial Research 2015, 1(1), 25-30.

27. Okoye, E. L., Uba, B. O., Uhobo, P. C., Oli, A. N., and Ikegbunam, M. N. (2014). Evaluation of the Antibacterial Activity of Methanol and Chloroform Extracts of Alchornea cordifolia Leaves. Journal of Scientific Research \& Reports, 3(June 2012), 255-262.

28. OMS. (2002). Stratégie de l'OMS pour la médecine traditionnelle pour 2002-2005. WHO/EDM/TRM.

29. OMS. (2011). Statistiques sanitaires mondiales. OMS.

30. OMS. (2013). Les maladies diarrhéiques : Aide-mémoire.

31. Ondo, J. P. (2011). Etude phytochimique et évaluation de l'activité antiplasmodiale de Vitex madiensis Oliv. (Lamiaceae) et de Croton mayumbensis (Euphorbiaceae), plantes médicinales gabonaises. Thèse. Université de Rouen.

32. Ondo, P. J., Obame, L. C., Andzi Barhé, T., Nsi Akoue, G., and Nsi Emvo, E. (2013). Phytochemical screening , total phenolic content and antiradical activity of Asplenium africanum ( Aspleniaceae ) and fruit of Megaphrinium macrostachyum (Marantaceae). Journal of Applied Pharmaceutical Science, 3(8), 92-96. https://doi.org/10.7324/JAPS.2013.3816

33. Paris, R., and Moyse, H. (1969). Précis de matière médicinale. In Paris : Masson (pp. 205-208).

34. Pincemail, J., Degrune, F., Voussure, S., and Malherbe, C. (2007). Effet d'une alimentation riche en fruits et légumes sur les taux plasmatiques en antioxydants et des marqueurs des dommages oxydatifs Effect of a diet rich in fruits and vegetables on the plasmatic antioxidant rates and of the markers of the oxidative d. Nutrition Clinique et Métabolisme, 21, 66-75. https://doi.org/10.1016/j.nupar.2007.04.005

35. Scherer, R., and Godoy, H. T. (2009). Antioxidant activity index (AAI) by 2,2-diphenyl-1- picrylhydrazyl method. Food Chem, 112, 654-658.

36. Shoji, T., Akazome, Y., Kanda, T., and Ikeda, M. (2004). The toxicology and safety of apple polyphenol extract. Food and Chemical Toxicology, 42, 959-967. https://doi.org/10.1016/j.fct.2004.02.008

37. Tene, M., Ndontsa, B. L., Tane, P., Tamokou, J. D. D., and Kuiate, J. R. (2009). Antimicrobial diterpenoids and triterpenoids from the stem bark of Croton macrostachys. Int. J. Biol. Chem. Sci, 3(3), 538-544.

38. Yala, J. F., Mabika Mabika, R., Camara, B., Tuo, S., Souza, A., Lepengue, N. A., and Mbatchi, B. (2017). Assessment of the antibacterial activity of four essential oils and the. International Journal of Phytomedicine, 9(3), 443-450. https://doi.org/10.5138/09750185.2106 
39. Yala, J. F., Ntsameso-Mve-Mba, V., Azzizet Issembe, Y., Lepengue, A. N., and Souza, A. (2016). Évaluation in vitro de 1' activité antimicrobienne de 1' extrait aqueux d' Eryngium foetidium récolté dans la ville de Franceville . Journal of Applied Biosciences, 103, 9886-9893.

40. Yang, Y. L., Li, S. Y., Cheng, H. H., and Lo, H. J. (2005). Susceptibilities to amphotericin B and fluconazole of Candida species in TSARY 2002. Diagn Microbiol Infect Dis, 51(3), 179-83.

41. Zongo, C., Savadoga, A., Ouattara, L., Bassole, H. N., Ouattara, C. A. T., Ouattar, A. S., and Traore, A. . (2010). Polyphenols Content, Antioxydant and Antimicrobial activities of Ampelocissus grantii (Baker) Planch. (Vitaceae): A Medicinal plant from Burkina Faso. Int Jof Pharm, 6(6), 880-887. 